\title{
Understanding the Effect of Skin Formation on the Removal of Solvents from Semicrystalline Polymers
}

\author{
SIM-SIONG WONG, ${ }^{1}$ SACIDE ALSOY ALTINKAYA, ${ }^{2}$ SURYA K. MALLAPRAGADA ${ }^{1}$ \\ ${ }^{1}$ Department of Chemical Engineering, lowa State University, 2114, Sweeney Hall, Ames, lowa 50011-2230 \\ ${ }^{2}$ Department of Chemical Engineering, Izmir Institute of Technology, Gulbahce Koyu, 35437 Urla-Izmir, Turkey
}

Received 25 March 2005; revised 20 June 2005; accepted 26 August 2005

DOI: 10.1002 / polb.20615

Published online in Wiley InterScience (www.interscience.wiley.com).

\begin{abstract}
The effect of glassy skin formation on the drying of semicrystalline polymers was investigated with a comprehensive mathematical model developed for multicomponent systems. Polymers with high glass-transition temperatures can become rubbery at room temperature under the influence of solvents. As the solvents are removed from the polymer, a glassy skin can form and continue to develop. The model takes into account the effects of diffusion-induced polymer crystallization as well as glassy-rubbery transitions on the overall solvent content and polymer crystallinity. A Vrentas-Duda free-volume-based diffusion scheme and crystallization kinetics were used in our model. The polymer-solvent system chosen was a poly(vinyl alcohol) (PVA)-water-methanol system. The drying kinetics of PVA films were obtained by gravimetric methods with swollen films with known water/methanol concentrations. The overall drying behaviors of the polymer system determined by our model and experimental methods were compared and found to match well.
\end{abstract}

() 2005 Wiley Periodicals, Inc. J Polym Sci Part B: Polym Phys 43: 3191-3204, 2005

Keywords: crystallization; drying; glass transition; modeling; poly(vinyl alcohol); semicrystalline polymers

\section{INTRODUCTION}

As the last process in polymer film production, polymer film drying plays an important role in controlling the structure and properties for various commercial products such as adhesive tapes, functional coatings, and photographic films., ${ }^{1,2}$ The microstructure of the polymer changes constantly during drying as the solvent is removed and the polymer chains realign themselves. ${ }^{3}$ In the design of drying processes, several issues need to be addressed, including the viscoelasticity of the polymer, the final solvent residual content in the film, and the structural integrity of the

Correspondence to: S. K. Mallapragada (E-mail: suryakm@ iastate.edu)

Journal of Polymer Science: Part B: Polymer Physics, Vol. 43, 3191-3204 (2005) (1) 2005 Wiley Periodicals, Inc. film. Because certain solvents are harmful to consumers, the polymer films produced must meet specific guidelines for residual solvent content. ${ }^{4-6}$ To maintain the structural integrity of the films during the course of the polymer shelf life, the films must be defect-free. ${ }^{7}$ Usually, various kinds of defects in polymer films are attributed to poorly chosen drying conditions. Thus, a clear understanding of the drying process is necessary for process design and optimization.

Although the behavior of amorphous polymers under various drying conditions has been studied, ${ }^{7-21}$ the drying characteristics of semicrystalline polymers are still lacking and are different from those of amorphous polymers. The removal of solvents from semicrystalline polymers opens a path for crystallization to occur because of voids during the drying process. The creation and growth of crystallites within the film 


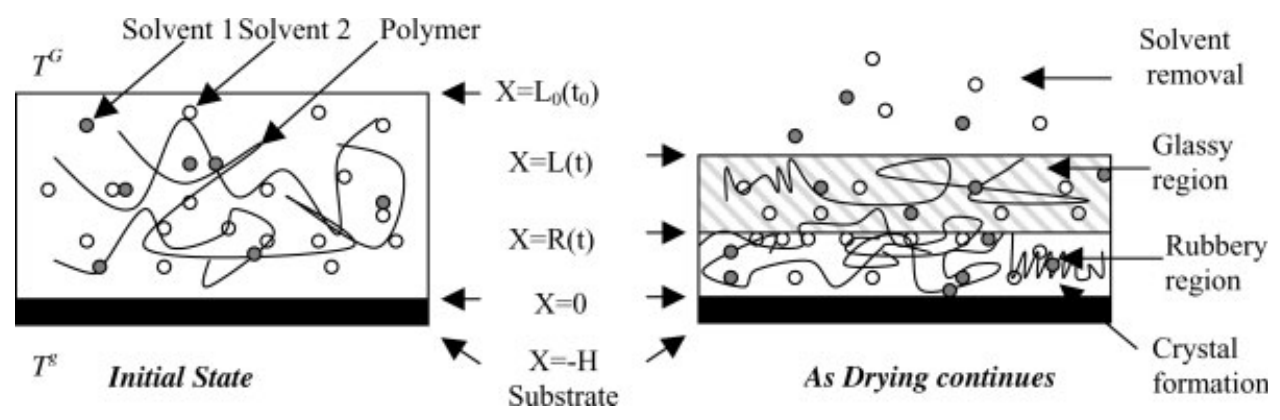

Figure 1. Schematic of semicrystalline polymer drying with the formation of a glassy skin.

will change the diffusion rates of solvents because solvent molecules need to diffuse around the crystals. ${ }^{22-24}$ In addition to the effects of crystallization, the glass-transition temperature of the polymer-solvent mixture also has an important role in the drying of polymer films. ${ }^{17,25-29}$ As solvents are removed, the glass-transition temperature of the mixture increases, and a glassy-rubbery transition may occur. Consequently, a glassy skin may develop that changes the rate of diffusion of solvents significantly. Previously, we developed a mathematical model to describe the drying behavior of rubbery semicrystalline polymers. ${ }^{3}$ The focus of this work is to extend our basic model to investigate the effect of the glassy-rubbery transition and glassy skin formation on the rate of removal of solvents from semicrystalline polymer-solvent mixtures.

\section{THEORY}

Several models have been proposed by various researchers investigating the behavior of amorphous polymers, glassy polymers, and semicrystalline polymers; each has distinct features and limitations. Our goal was to develop a comprehensive, broad-based model to better understand the interconnectivity of phenomena such as crystallization and glass transition that affect drying. Figure 1 shows a schematic of the drying process represented in our model. The polymer-solventsolvent system was placed on an impermeable substrate. The initial thickness of the film was $L_{0}$, and the thickness of the substrate, $H$, was constant. This polymer-substrate system was then subjected to hot air on the top and bottom sides with temperatures $T^{\mathrm{G}}$ and $T^{\mathrm{g}}$, respectively. As drying continued, the overall film thickness, $L(t)$, began to decrease as the solvents evaporated out of the system. At the same time, a glassy skin was assumed to form at the exposed polymer-air surface, thus effectively creating a two-region system: a glassy region near the polymer-air interface and a rubbery region underneath it. Inside the glassy region, drying-induced crystallization was assumed to cease because of the decrease in polymer mobility and the significant increase in the diffusional resistances of the solvents. In the rubbery region, crystal formation was governed by polymer chain mobility that can be described by the free-volume theory. This kinetic model was developed by Ramesh ${ }^{30}$ and takes into account the plasticizing ability of the solvents through free-volume parameters. Ramesh proposed that crystal formation is proportional to a rate constant that is a function of the amount of the solvent and the difference between the crystallinity at any time and that at $t \rightarrow \infty$. We have extended this binary kinetic model to a ternary system. In our drying model, the mass transfer was assumed to be governed by the diffusion of solvents and the crystallizationinduced convection when the amorphous phase was transformed into a crystalline phase.

In our model, one-dimensional transport was assumed because the thickness of the polymer film was much smaller than the other dimensions of the film. In the formulation of mass-transfer and kinetic equations, volume fractions of the polymer and solvents were used. The complexity arising from density differences between the polymer and solvents was eliminated by the assumption of no volume change upon mixing. The polymer system was composed of four components represented by $N$ : solvent 1 , solvent 2 , amorphous polymer, and crystalline polymer. The subscripts 1 through 4 represent these components, respectively. In our equations, $w_{1}$ and $w_{2}$ represent the volume fractions of solvents 1 and 2 , whereas the volume fractions of the amorphous and crystalline polymer regions are denoted by $u$ and $v$. The 
development of glassy skin in the polymer system effectively creates two different diffusion regions: a shrinking rubbery region with a high solventdiffusion rate and a growing glassy region with a limited solvent-diffusion rate. Thus, the calculation of the solvent concentrations in each region was performed separately. This created a moving boundary problem with two different moving fronts: the polymer-air interface and the glassyrubbery interface.

In the rubbery region, the species continuity equations for the solvents were written as follows:

$$
\frac{\partial w_{i}}{\partial t}+\frac{\partial}{\partial x}\left(w_{i} v^{\neq}\right)=\frac{\partial}{\partial x}\left(\sum_{j=1}^{N-2} D_{i j}^{R} \frac{\hat{V}_{i}}{\hat{V}_{j}} \frac{\partial w_{j}}{\partial x}\right)
$$

Here $w_{i}$ represents the volume fraction of component $i, v^{\neq}$is the volume average velocity generated by the convection due to crystallization, $\hat{V}_{i}$ represents the partial specific volume of component $i$, and $D_{i j}^{\mathrm{R}}$ represents the main diffusion and cross-diffusion coefficients in the rubbery region. The volume average velocity can be calculated as follows:

$$
\frac{\partial v^{f}}{\partial x}=r_{c}\left(\hat{V}_{3}-\hat{V}_{4}\right)
$$

where $r_{\mathrm{c}}$ is the rate of crystallization, $\hat{V}_{4}$ is the specific volume of the crystalline polymer, and $\hat{V}_{3}$ is the specific volume of the amorphous polymer. $r_{\mathrm{c}}$ can be expressed as a function of the current, $v$, and the final crystalline polymer volume fraction, $v_{\infty}$, as follows:

$$
r_{c}=-k \frac{v}{\hat{V}_{4}}\left(v-v_{\infty}\right)
$$

where the rate constant, $k$, defined in eq 4 is a function of the mobility of the amorphous phase and is calculated from the Vrentas-Duda free-volume theory: ${ }^{31,32}$

$$
k=k_{0} \exp \left(\frac{\frac{\omega_{1} \hat{V}_{1}^{*}}{\xi_{13}^{R}}+\frac{\omega_{2} \hat{V}_{2}^{*}}{\xi_{23}^{R}}+\omega_{3} \hat{V}_{3}^{*}}{\frac{\hat{V}_{\mathrm{FH}}}{\gamma}}\right)
$$

In eq $4, \omega_{i}$ is the weight fraction of component $i$ and $\hat{V}_{i}^{*}, \xi_{i j}^{\mathrm{R}}$, and $\hat{V}_{F H} / \gamma$ are the free-volume parameters. A discussion of the Vrentas-Duda free-volume theory can be found in a later section. The volume fraction of the crystalline polymer was determined by eq 5 :

$$
\frac{\partial v}{\partial t}=r_{c} \hat{V}_{4}
$$

In our equations, the flux of the amorphous polymer is excluded. However, this does not mean that its contribution is neglected. The starting point of these equations includes the gradients of the chemical potential of each species based on Bearman statistical mechanical theory, ${ }^{33}$ as a result of which the flux of one of the components, usually the polymer, is eliminated. With the use of volume fractions, the volume fraction of the amorphous polymer is implicitly dependent on the volume fractions of the other components and is calculated with the following equation:

$$
u=1-w_{1}-w_{2}-v
$$

For the glassy region of the polymer, similar continuity equations were written:

$$
\frac{\partial w_{i}}{\partial t}=\frac{\partial}{\partial x}\left(\sum_{j=1}^{N-2} D_{i j}^{G} \frac{\hat{V}_{i}}{\hat{V}_{j}} \frac{\partial w_{j}}{\partial x}\right)
$$

Because we assumed that the polymer does not crystallize in the glassy region on account of chain immobility, the convective term arising from crystallization was eliminated. The diffusion scheme used here was the same as that described in the rubbery region.

No mass transfer was assumed to take place at the polymer-substrate boundary:

$$
\frac{\partial w_{i}}{\partial x}=0
$$

At the glassy-rubbery interface, the mass fluxes of the solvents were continuous:

$$
\begin{aligned}
-\frac{D_{i 1}^{R}}{\hat{V}_{1}} \frac{\partial w_{1}}{\partial x}-\frac{D_{i 2}^{R}}{\hat{V}_{2}} \frac{\partial w_{2}}{\partial x} & -\frac{w_{i}}{\hat{V}_{i}} \frac{d R}{d t}+\frac{w_{i}}{\hat{V}_{i}} v^{\neq} \\
& =-\frac{D_{i 1}^{G}}{\hat{V}_{1}} \frac{\partial w_{1}}{\partial x}-\frac{D_{i 2}^{G}}{\hat{V}_{2}} \frac{\partial w_{2}}{\partial x}
\end{aligned}
$$

Similarly, an appropriate boundary condition at the polymer-gas interface was derived from the fact that mass fluxes were continuous in the polymer and the gas phases:

$$
-\frac{D_{i 1}^{G}}{\hat{V}_{1}} \frac{\partial w_{1}}{\partial x}-\frac{D_{i 2}^{G}}{\hat{V}_{2}} \frac{\partial w_{2}}{\partial x}-\frac{w_{i}}{\hat{V}_{i}} \frac{d L}{d t}=k_{i}^{m}\left(P_{i}^{i}-P_{i}^{b}\right)
$$


where $k_{i}^{\mathrm{m}}$ is the mass-transfer coefficient of species $i, P_{i}^{\mathrm{i}}$ is the interfacial partial pressure of species $i$, and $P_{i}^{\mathrm{b}}$ is the bulk partial pressure of species $i$. To calculate the rate of film shrinkage, a volume balance was used by the measurement of the amount of solvent loss due to evaporation at the interface:

$$
\frac{d L}{d t}=-\sum_{i=1}^{N-2} k_{i}^{m} \hat{V}_{i}\left(P_{i}^{i}-P_{i}^{b}\right)
$$

A simple $n$-order kinetics ${ }^{9,34}$ was assumed to describe the movement of the glassy-rubbery interface:

$$
\frac{d R}{d t}=-\sum_{i=1}^{N-2} a\left(w_{i}\right)^{b}
$$

where $a$ and $b$ are the kinetics constants. A single uniform temperature for the polymer film and the substrate layer was assumed because the convective resistance to heat transfer in the gas phase is much greater than the conductive resistance in the polymer and substrate layers. Thus, the overall temperature of polymer substrate is calculated with eq 13 :

$$
\frac{d T}{d t}=-\left[\frac{h^{G}\left(T-T^{G}\right)+h^{g}\left(T-T^{g}\right)+\sum_{i=1}^{N-2} k_{i}^{m} \Delta \hat{H}_{i}\left(P_{i}^{i}-P_{i}^{b}\right)+r_{c} \Delta H_{c r y s} R(t)}{\rho^{p} \hat{C}_{p}^{p} L(t)+\rho^{s} \hat{C}_{p}^{s} H}\right]
$$

The terms $h^{\mathrm{G}}, T^{\mathrm{G}}, h^{\mathrm{g}}$, and $T^{g}$ refer to the heattransfer coefficients and temperature for the top and bottom sides, respectively, $\Delta \hat{H}_{\mathrm{i}}$ refers to the latent heat of vaporization, $\Delta H_{\text {crys }}$ is the heat of crystallization, and $\hat{C}_{\mathrm{p}}$ is the heat capacity.

A series of four diffusion coefficients were calculated from a model developed by Alsoy and $\operatorname{Duda}^{1}$ and are given in eqs $14-17$ :

$$
D_{11}=D_{1} \rho_{1}\left(1-\rho_{1} \hat{V}_{1}\right) \frac{1}{R T} \frac{\partial \mu_{1}}{\partial \rho_{1}}-D_{2} \rho_{1} \rho_{2} \hat{V}_{2} \frac{1}{R T} \frac{\partial \mu_{2}}{\partial \rho_{1}}
$$

$$
D_{12}=D_{1} \rho_{1}\left(1-\rho_{1} \hat{V}_{1}\right) \frac{1}{R T} \frac{\partial \mu_{1}}{\partial \rho_{2}}-D_{2} \rho_{1} \rho_{2} \hat{V}_{2} \frac{1}{R T} \frac{\partial \mu_{2}}{\partial \rho_{2}}
$$

$$
D_{21}=D_{2} \rho_{2}\left(1-\rho_{2} \hat{V}_{2}\right) \frac{1}{R T} \frac{\partial \mu_{2}}{\partial \rho_{1}}-D_{1} \rho_{1} \rho_{2} \hat{V}_{1} \frac{1}{R T} \frac{\partial \mu_{1}}{\partial \rho_{1}}
$$

$$
D_{22}=D_{2} \frac{w_{2}}{\hat{V}_{2}}\left(1-w_{2}\right) \frac{1}{R T} \frac{\partial \mu_{2}}{\partial \rho_{2}}-D_{1} \frac{w_{2}}{\hat{V}_{2}} w_{1} \frac{1}{R T} \frac{\partial \mu_{1}}{\partial \rho_{2}}
$$

In these equations, $D_{11}$ and $D_{22}$ represent the main diffusion terms, whereas $D_{12}$ and $D_{21}$ are the cross-diffusion terms. $D_{1}$ and $D_{2}$ are the self-diffusion coefficients, and $(1 / R T)\left(\partial \mu_{i} / \partial \rho_{j}\right)$ is the thermodynamic contribution to the diffusion process, as determined by ternary FloryHuggins theory. ${ }^{35,36}$ Self-diffusion coefficients were obtained from the Vrentas-Duda free-volume theory, as shown in eqs $18-20:^{31,32}$

$D_{1}=D_{01} \exp \left[-\frac{E_{1}}{R T}\right] \exp \left[-\frac{\omega_{1} \hat{V}_{1}^{*}+\omega_{2} \hat{V}_{2}^{*} \frac{\xi_{13}}{\xi_{23}}+\omega_{3} \hat{V}_{3}^{*} \xi_{13}}{\frac{\hat{V}_{\mathrm{FH}}}{\gamma}}\right]$

$D_{2}=D_{02} \exp \left[-\frac{E_{2}}{R T}\right] \exp \left[-\frac{\omega_{1} \hat{V}_{1}^{*} \xi_{23}+\omega_{2} \hat{V}_{2}^{*}+\omega_{3} \hat{V}_{3}^{*} \xi_{23}}{\frac{\hat{V}_{\mathrm{FH}}}{\gamma}}\right]$

$$
\begin{aligned}
\frac{\hat{V}_{\mathrm{FH}}}{\gamma} & =\omega_{1} \frac{K_{11}}{\gamma}\left(K_{21}-T_{g 1}+T\right) \\
& +\omega_{2} \frac{K_{12}}{\gamma}\left(K_{22}-T_{g 2}+T\right)+\omega_{3} \frac{K_{13}}{\gamma}\left(K_{23}-T_{g 3}+T\right)
\end{aligned}
$$

A detailed description of several parameters and procedures for calculating these parameters can be found elsewhere. ${ }^{1,3,37-39}$ The free-volume parameters used in the glassy and rubbery regions 
are different, resulting in different diffusion coefficients in these regions. Free-volume parameters in the glassy region were estimated on the basis of the values obtained for the rubbery region of the same polymer.

In our system, there are two moving boundaries: the shrinking of the film and the development and movement of the glassy-rubbery interface. The movements of these boundaries makes the problem-solving techniques needed to include those effects more complicated. In our case, we employed a front-fixing method, in which these boundaries are fixed in a special coordinate system, where the glassy-rubbery interface is always at the location $\xi=1$ and the gas-polymer interface is always at the location $\xi=2$. The method that we chose is based on Landau transform. ${ }^{40}$ The spatial coordinate transformation is as follows:

$$
\begin{gathered}
\xi=\frac{x}{R(t)}, \quad 0 \leq \xi \leq 1 \\
\xi=1+\frac{x-R(t)}{L(t)-R(t)}, \quad 1 \leq \xi \leq 2
\end{gathered}
$$

where $x$ and $\xi$ represent the old and new space coordinates, respectively, and $L(t)$ and $R(t)$ represent the time-dependent positions of the boundaries, which in our case are the overall film thickness and the glassy-rubbery interface at any given time. Figure 2 shows the transformation of the coordinate. On the basis of these two equations, all the equations mentioned previously were transformed from $f(x, t)$ to $f(\xi, t)$ before being solved by finite-element numerical methods. With this new coordinate system, a variable-sized grid was developed with a finer mesh near the polymer-substrate interface, glassyrubbery interface, and gas-polymer interface. This is because the gradient near these interfaces is steep and changes from time step to time step, and this grid was designed to ensure accu- rate calculation while reducing the computational resources needed to solve the system. A finite-element method with MATLAB (MathWorks, Natick, MA) and FEMLAB (Comsol, Burlington, MA) software was used.

\section{EXPERIMENTAL}

\section{Materials}

Poly(vinyl alcohol) (PVA) powders with molecular weights of 64,000 (Elvanol grades, E. I. du Pont de Nemours, Wilmington, DE) and 133,000 (Polysciences, Warrington, PA) were used. Both PVA samples were fully hydrolyzed (degree of hydrolysis $>99.0 \%$ ) with polydispersity indices of 2.15 . PVA films were made by the dissolution of the PVA powder in water and casting onto siliconized Petri dishes. The films were dried until a constant weight was achieved.

\section{Crystallinity of the Films}

The final crystalline contents of the films were determined with differential scanning calorimetry (DSC; DSC7, PerkinElmer, Boston, MA). A small sample of PVA was heated from 25 to $250{ }^{\circ} \mathrm{C}$ at $10{ }^{\circ} \mathrm{C} / \mathrm{min}$, and the melting profile was recorded. The latent heat of melting $(\Delta H)$ value measured was compared to the $\Delta H$ value of pure PVA crystals $(138.6 \mathrm{~J} / \mathrm{g}){ }^{41}$

\section{Drying Experiments}

Dried films were swollen in methanol and water separately to achieve the desired initial water/ methanol concentration. The weight of each film was monitored throughout the swelling process to determine the solvent uptake. Possible cross-contamination of solvents has been addressed previously, and we found no significant drawback to this method. Thermogravimetric analysis (TGA7, PerkinElmer) was used to monitor the total weight

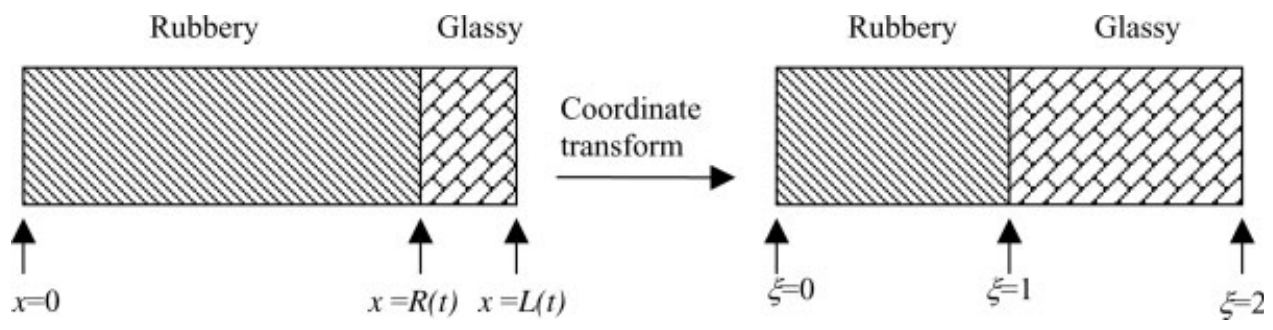

Figure 2. Schematic of the transformation to a new fixed spatial coordinate for solving the system with a numerical method. 
Table 1. Free-Volume Parameters Used in Mathematical Modeling for PVA-Water (Solvent 1)-Methanol (Solvent 2) ${ }^{\mathrm{a}}$

\begin{tabular}{|c|c|c|c|c|}
\hline \multirow[b]{2}{*}{ Parameter } & \multicolumn{2}{|c|}{ PVA-Water } & \multicolumn{2}{|c|}{ PVA-Methanol } \\
\hline & Glassy & Rubbery & Glassy & Rubbery \\
\hline$D_{o i} \mathrm{~cm}^{2} \mathrm{~s}^{-1}$ & 0.941 & 0.705 & 0.00155 & 0.00116 \\
\hline$E_{l} \mathrm{~J} \mathrm{~mol}^{-1}$ & 7978 & 7978 & -3585 & 3585 \\
\hline$K_{11} / \gamma \mathrm{cm}^{3} \mathrm{~g}^{-1} \mathrm{~K}^{-1}$ & $1.65 \times 10^{-3}$ & $1.65 \times 10^{-3}$ & - & - \\
\hline$K_{12} / \gamma \mathrm{cm}^{3} \mathrm{~g}^{-1} \mathrm{~K}^{-1}$ & - & - & $5.64 \times 10^{-4}$ & $5.64 \times 10^{-4}$ \\
\hline$K_{13} / \gamma \mathrm{cm}^{3} \mathrm{~g}^{-1} \mathrm{~K}^{-1}$ & $2.29 \times 10^{-4}$ & $2.29 \times 10^{-4}$ & $2.29 \times 10^{-4}$ & $2.29 \times 10^{-4}$ \\
\hline$K_{21} \mathrm{~K}$ & -141.73 & -141.73 & - & - \\
\hline$K_{22} \mathrm{~K}$ & - & - & 23.87 & 23.87 \\
\hline$K_{23} \mathrm{~K}$ & -214.87 & -214.87 & -214.87 & -214.87 \\
\hline$T_{g 1} \mathrm{~K}$ & 0 & 0 & - & - \\
\hline$T_{g 2} \mathrm{~K}$ & - & - & 0 & 0 \\
\hline$T_{g 3} \mathrm{~K}$ & 0 & 0 & 0 & 0 \\
\hline$V_{1}^{*} \mathrm{~cm}^{3} \mathrm{~g}^{-1}$ & 1.071 & 1.071 & - & - \\
\hline$V_{2}^{*} \mathrm{~cm}^{3} \mathrm{~g}^{-1}$ & - & - & 0.959 & 0.959 \\
\hline$V_{3}^{*} \mathrm{~cm}^{3} \mathrm{~g}^{-1}$ & 0.720 & 0.720 & 0.720 & 0.720 \\
\hline$\xi_{i j}$ & 0.45 & 0.45 & 0.99 & 0.99 \\
\hline$\chi_{i 3}$ & 0.67 & 0.67 & 1.26 & 1.26 \\
\hline$\chi_{12}$ & 0.442 & 0.442 & 0.442 & 0.442 \\
\hline
\end{tabular}

${ }^{\text {a }}$ Data taken from ref. 42.

of the film during the course of the drying. The film was dried at $30{ }^{\circ} \mathrm{C}$ with air flow from the top side of $25 \mathrm{~mL} / \mathrm{min}$ for $1 \mathrm{~h}$.

\section{RESULTS AND DISCUSSION}

Table 1 lists the free-volume parameters for the PVA-water-methanol system. These parameters were obtained independently from diffusion-temperature and viscosity-temperature data. From experimental studies, the final crystallinity of the polymer films was found to be approximately
$40 \%$. The physical properties of the system and the operating conditions used in the simulations are listed in Tables 2 and 3. The model predictions based on these parameters are shown in Figures 3-6. The volume fractions of water in the rubbery and glassy regions are shown in Figure 3. Although the overall thickness of the glassy and rubbery regions changed continuously throughout the drying process, the volume fractions are plotted in fixed spatial coordinates normalized with the interfaces, as discussed previously. In Figures 3 and $4, \xi=0$ and $\xi=1$ represent the substraterubbery and glassy-rubbery interfaces, respec-

Table 2. Properties of the Polymer System ${ }^{\mathrm{a}}$

Film Properties

Heat capacity, $\hat{C}_{\mathrm{p}}^{\mathrm{p}}$

Density of the film, $\rho^{\mathrm{p}}$

Heat of vaporization of water, $\Delta \hat{H}_{1}$

Heat of vaporization of methanol, $\Delta \hat{H}_{2}$

Crystallization kinetic coefficient, $k_{0}$

Parameter for the glassy-rubbery boundary, $a$

Parameter for the glassy-rubbery boundary, $b$

Substrate Properties

Heat capacity, $\hat{C}_{\mathrm{p}}^{\mathrm{s}}$

Density of the substrate, $\rho^{\mathrm{s}}$

Substrate thickness, $H$

$$
\begin{aligned}
& 1.674 \mathrm{~J} \mathrm{~g}^{-1} \mathrm{~K}^{-1} \\
& 1.294 \mathrm{~g} \mathrm{~cm}^{-3} \\
& 2404 \mathrm{~J} \mathrm{~g}^{-1} \\
& 1155 \mathrm{~J} \mathrm{~g}^{-1} \\
& 2 \times 10^{-6} \mathrm{~s}^{-1} \\
& 5.8 \times 10^{-5} \\
& 2 \\
& \\
& 0.84 \mathrm{~J} \mathrm{~g}^{-1} \mathrm{~K}^{-1} \\
& 2.6 \mathrm{~g} \mathrm{~cm}^{-3} \\
& 0.012 \mathrm{~cm}^{-1}
\end{aligned}
$$

\footnotetext{
${ }^{\text {a }}$ Data taken from refs. 43 and 44
} 
Table 3. Initial and Boundary Conditions for the PVA-Water-Methanol System for the Simulation Test ${ }^{\mathrm{a}}$

Initial Conditions

Temperature, $T_{0}$

Film thickness, $L_{0}$

Initial volume fraction of solvent $1, w_{10}$

Initial volume fraction of solvent $2, w_{20}$

Initial volume fraction of the crystalline polymer, $v_{0}$

Initial volume fraction of the amorphous polymer, $u_{0}$

Operating Conditions

Gas-polymer heat-transfer coefficient, $h^{\mathrm{G}}$

Gas-substrate heat-transfer coefficient, $h^{\mathrm{g}}$

Top-side air temperature, $T^{\mathrm{G}}$

Bottom-side air temperature, $T^{\mathrm{g}}$

Solvent 1 mass-transfer coefficient, $k_{i}^{\mathrm{G}}$

Solvent 2 mass-transfer coefficient, $k_{l}^{\mathrm{g}}$

Molar fraction of solvent 1 in gas

Molar fraction of solvent 2 in gas
$298 \mathrm{~K}$
$0.5 \mathrm{~mm}$
0.6
0.15
0.01
0.24

$0.029 \mathrm{~W} \mathrm{~cm}^{-2} \mathrm{~K}^{-1}$

$0.012 \mathrm{~W} \mathrm{~cm}^{-2} \mathrm{~K}^{-1}$

$303 \mathrm{~K}$

$303 \mathrm{~K}$

$2.07 \times 10^{-10} \mathrm{~s} \mathrm{~cm}^{-1}$

$1.67 \times 10^{-14} \mathrm{~s} \mathrm{~cm}^{-1}$

0

0

${ }^{\text {a }}$ Data taken from refs. 43 and 44.

tively. This region is established as the rubbery region. The glassy region spans from $\xi=1$ to $\xi$ $=2$, which corresponds to the glassy-region/air interface. The relatively flat profiles shown in Figure 3 in the rubbery region suggest that diffusive resistance was small. Furthermore, the pro- files in this region remained similar throughout the drying process. At the glassy-rubbery interface, $\xi=1$, a concentration gradient developed, revealing a barrier formed that restricted the movement of water. In the glassy region, the volume fraction profiles of water look different. Com-

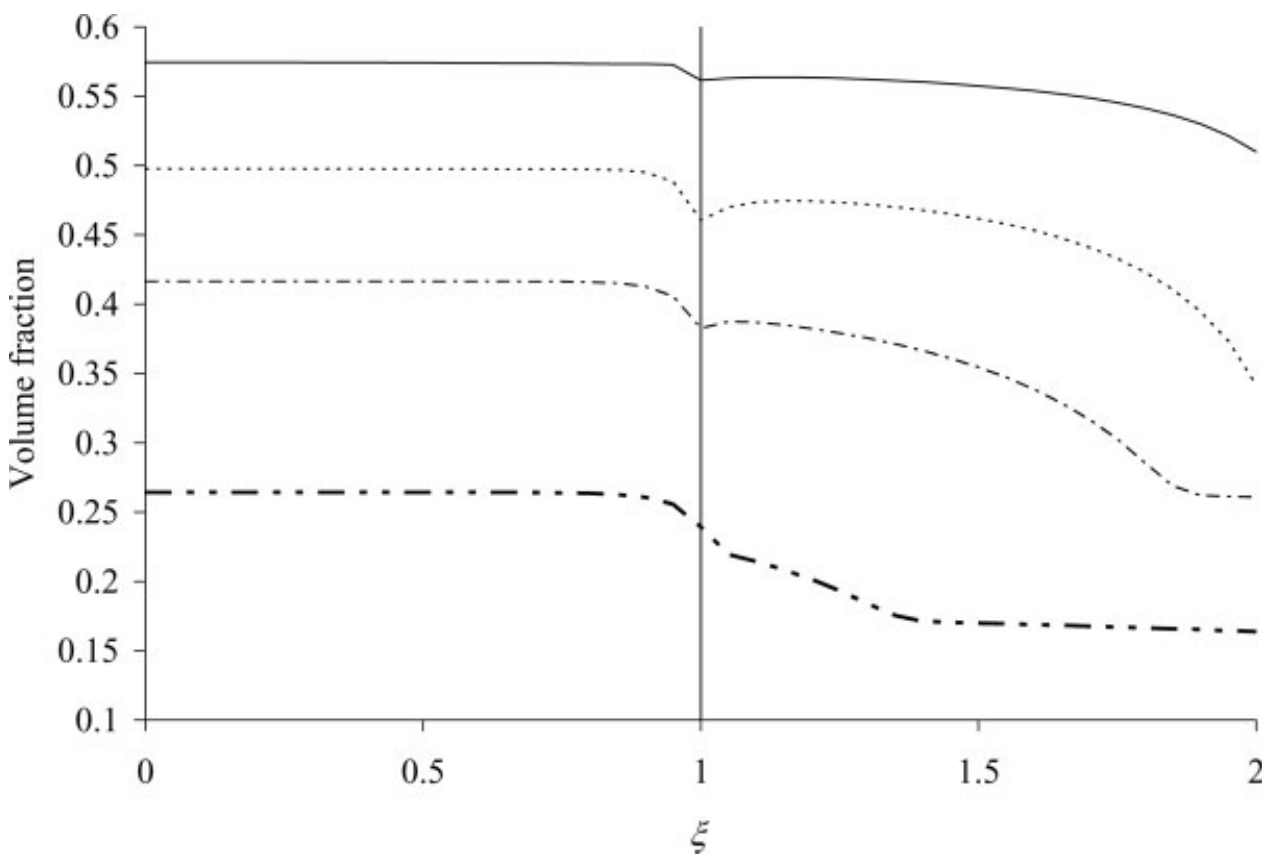

Figure 3. Volume fraction of water in the rubbery and glassy regions during drying: (-) the volume fraction profile at $5 \mathrm{~min}$ into drying, (. . .) the volume fraction profile at $15 \mathrm{~min}$ into drying, $(\cdot-\cdot)$ the volume fraction profile at $25 \mathrm{~min}$ into drying, and (....) the volume fraction profile at $45 \mathrm{~min}$ into drying. The rubbery region spans from $\xi=0$ to $\xi=1$, and the glassy region spans from $\xi=1$ to $\xi=2$. The glassy-rubbery interface is located at $\xi=1$. 


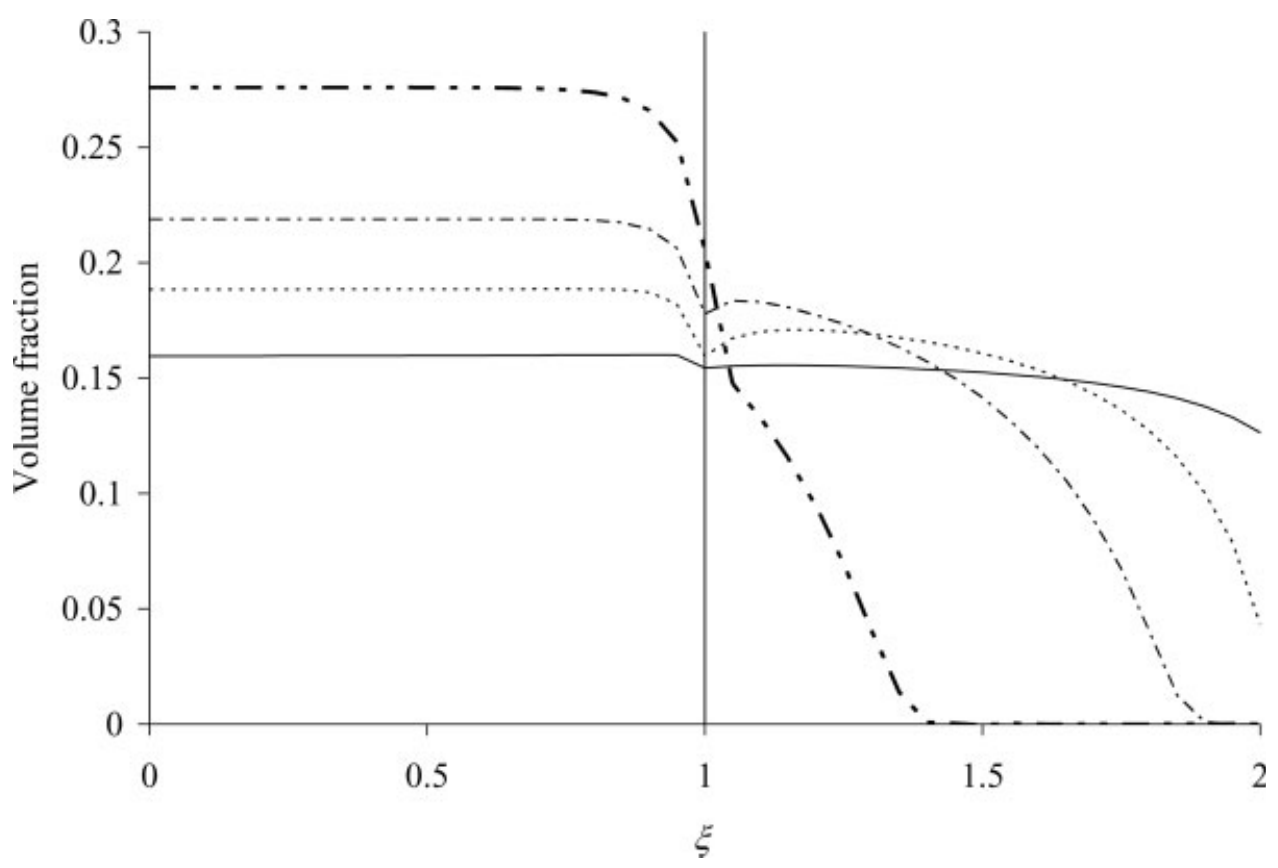

Figure 4. Volume fraction of methanol in the rubbery and glassy regions: (-) the volume fraction profile at $5 \mathrm{~min}$ into drying, (. .) the volume fraction profile at 15 min into drying, $(.-$.$) the volume fraction profile at 25$ min into drying, and (. . - .) the volume fraction profile at $45 \mathrm{~min}$ into drying. The rubbery region spans from $\xi=0$ to $\xi=1$, and the glassy region spans from $\xi=1$ to $\xi=2$. The glassy-rubbery interface is located at $\xi=1$.

pared with that of the rubbery region, the diffusion resistance in this region was larger, as expected. In addition, the shape of the profiles changed as drying continued. We attribute this change in shape to the development of the barrier in the glassy-rubbery transition area. A shift

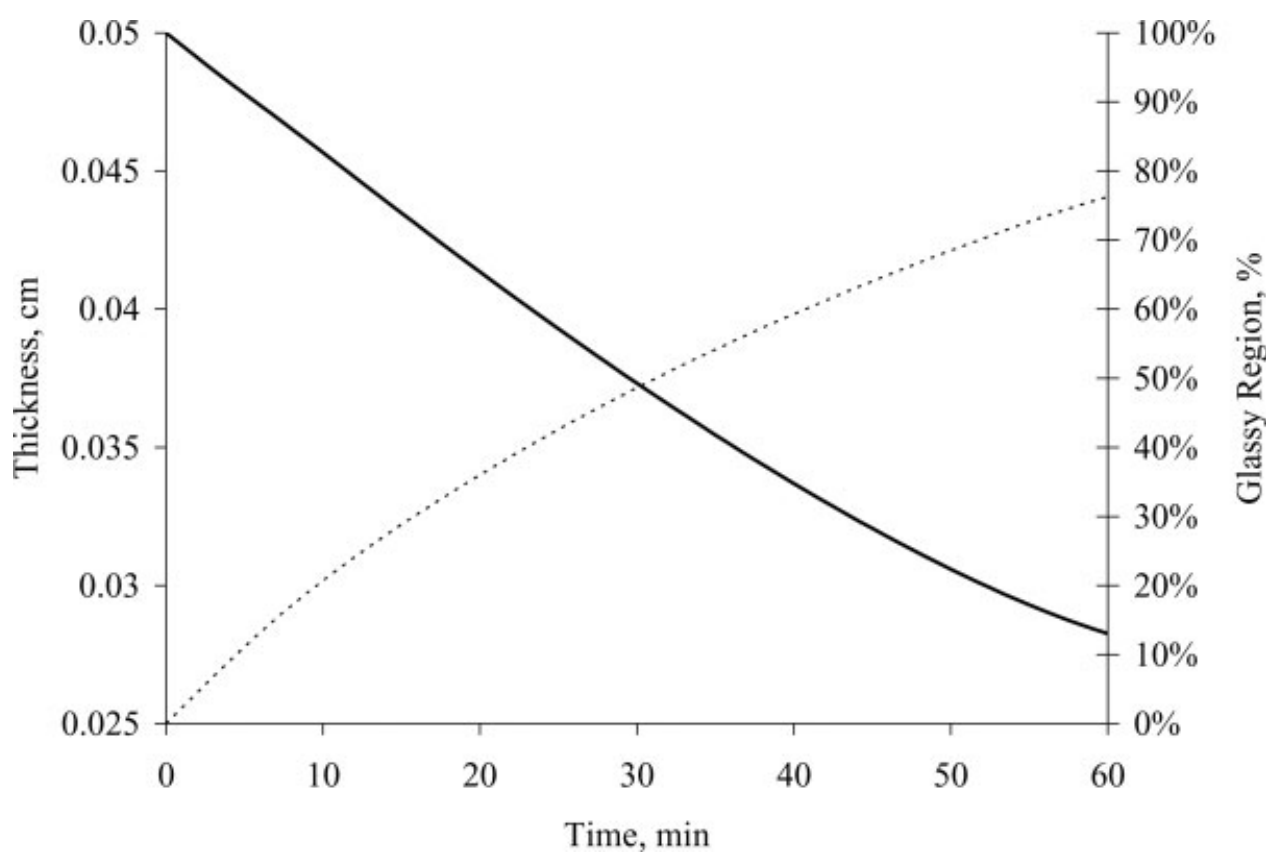

Figure 5. Changes in (-) the thickness of the overall film and (...) the thickness of the glassy region in the system as the drying continues. 
in the diffusive resistance is shown in Figure 3 (glassy region), in which the steepest gradient changed from the polymer-gas interface $(\xi=2)$ to the glassy-rubbery interface $(\xi=1)$ with time. Similar profiles were observed for the volume fraction of methanol within each region, as shown in Figures 4. In the rubbery region of Figure 4, an increase in the volume fraction of methanol was observed because of the fast removal of water. This phenomenon has been observed elsewhere. ${ }^{1}$ Also, solvent trapping occurred in the rubbery region as the glassy region developed. Figure 5 shows the change in the thickness of the film as drying progressed along with the development of the glassy region in the system during drying. The development of a glassy skin occurred as the drying started and continued to move across the depth of the film as drying continued. In Figure $6(\mathrm{a}-\mathrm{c})$, the average volume fractions of the water, methanol, and crystalline region are shown. In Figure 6(a), a steady decrease of the average volume fraction of water can be observed, as the diffusional resistance within the film is low and water removal is mostly determined by the external mass-transfer rate. In Figure 6(b), the average volume fraction of methanol goes through a maximum before decreasing as drying continues. This is due to the rapid removal of water, as shown in Figure 6(a), causing the overall volume of the system to drop faster than the rate of methanol removal, thus increasing the volume fraction of methanol in the system. Figure 6(c) shows the average volume fraction of the crystalline polymer in the rubbery region during drying. In the rubbery region, crystal formation and growth were dictated by eq 6 . As the drying continued, the average volume fraction of the crystals increased until reaching a predetermined value, which was the final crystallinity of the film. The crystallinity of a dried PVA film was determined with DSC results and literature values. ${ }^{41}$ As shown in Figure 6(c), the growth of the crystalline phase was rapid during the early stages of the drying, driven by the available free volume created by the removal of solvents. As it approached the final crystallinity, the growth process slowed and plateaued after $15 \mathrm{~min}$ of drying. In the glassy region, no crystallization occurred because of chain immobility. However, as the glassy-rubbery boundary pushed inward deeply into the film, crystals that had already formed in the rubbery region were included in the glassy region along with the amorphous phase of the polymer. Thus, the overall volume fraction of

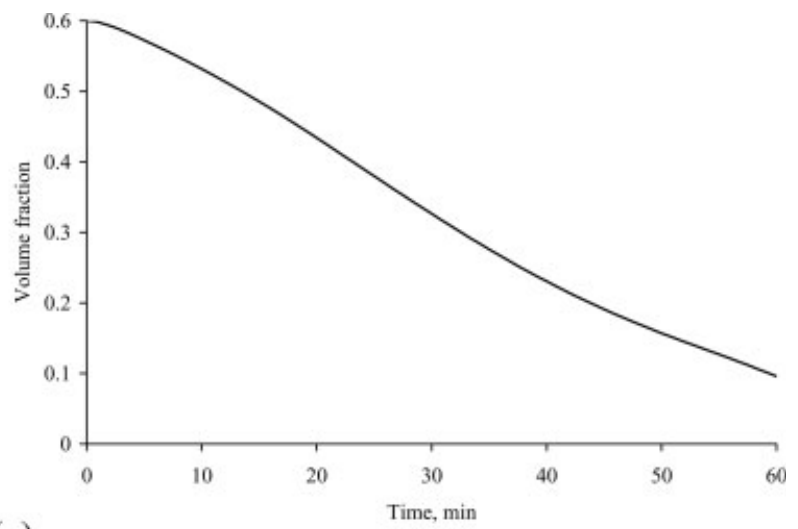

(a)

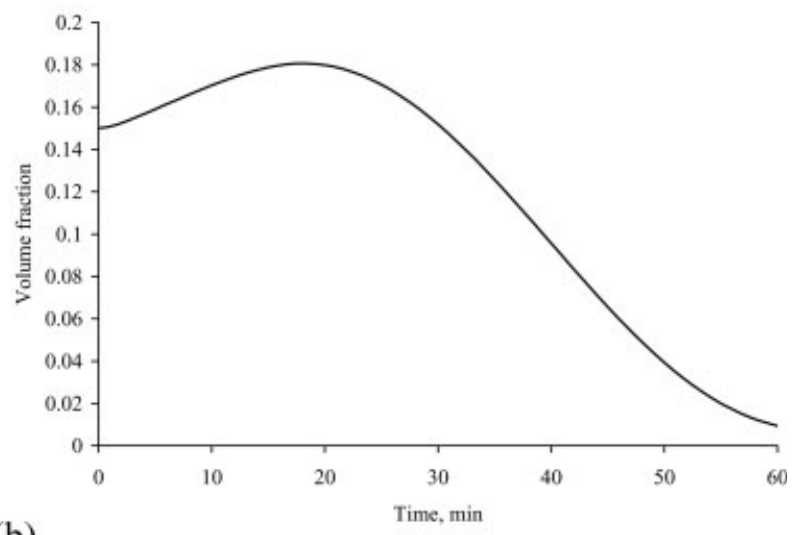

(b)

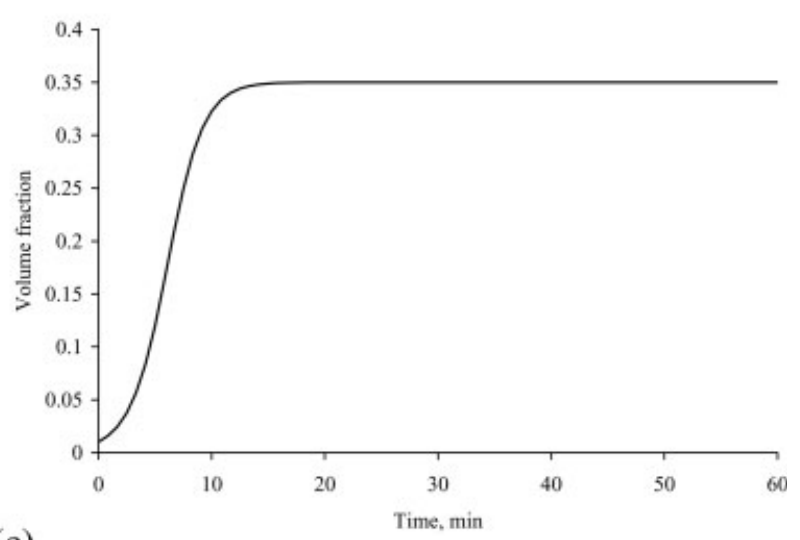

(c)

Figure 6. Average volume fraction of (a) water, (b) methanol, and (c) crystalline polymer during drying.

the crystalline polymer increased as drying continued. This increase was directly proportional to the amount of crystals at the glassy-rubbery interface and the rate of growth of the glassy-rubbery boundary.

The effects of temperature on the drying behavior of PVA were studied at two different air temperatures $\left(30\right.$ and $35{ }^{\circ} \mathrm{C}$ ), while other parameters, as listed in Tables 1-3, were held constant. 


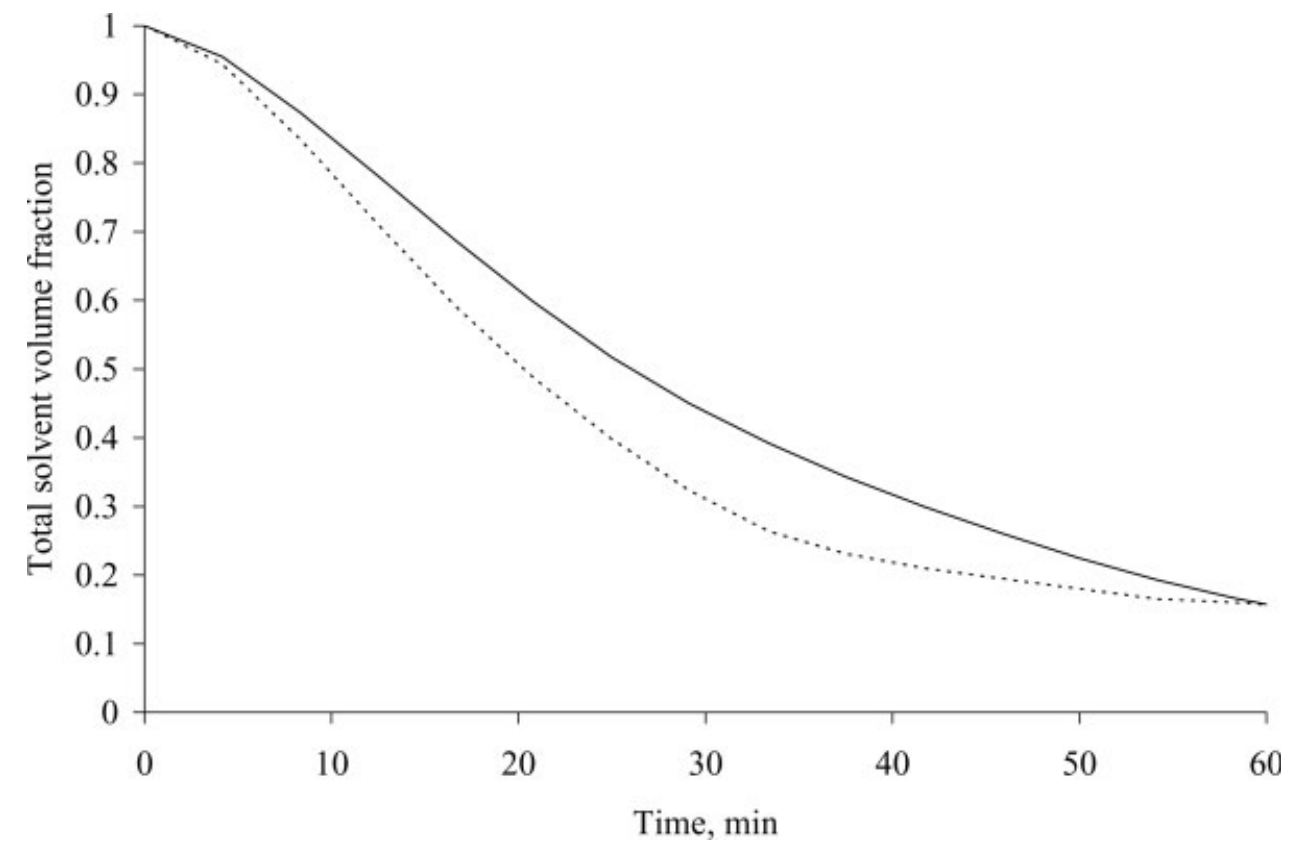

Figure 7. Total residual solvent volume fraction at different temperatures: (-) the remaining solvent volume fraction when drying is performed at $30{ }^{\circ} \mathrm{C}$ and $(\cdots)$ the volume fraction when the system is dried at $35{ }^{\circ} \mathrm{C}$.

The results are shown in Figure 7. The increase in the operating temperature increased the activity of the solvents, thus increasing the initial rate of solvent removal. However, small increases in temperature did not have a large effect on the overall solvent residual concentration within our experimental timescale because of the high diffusional resistance within the film at that stage. To

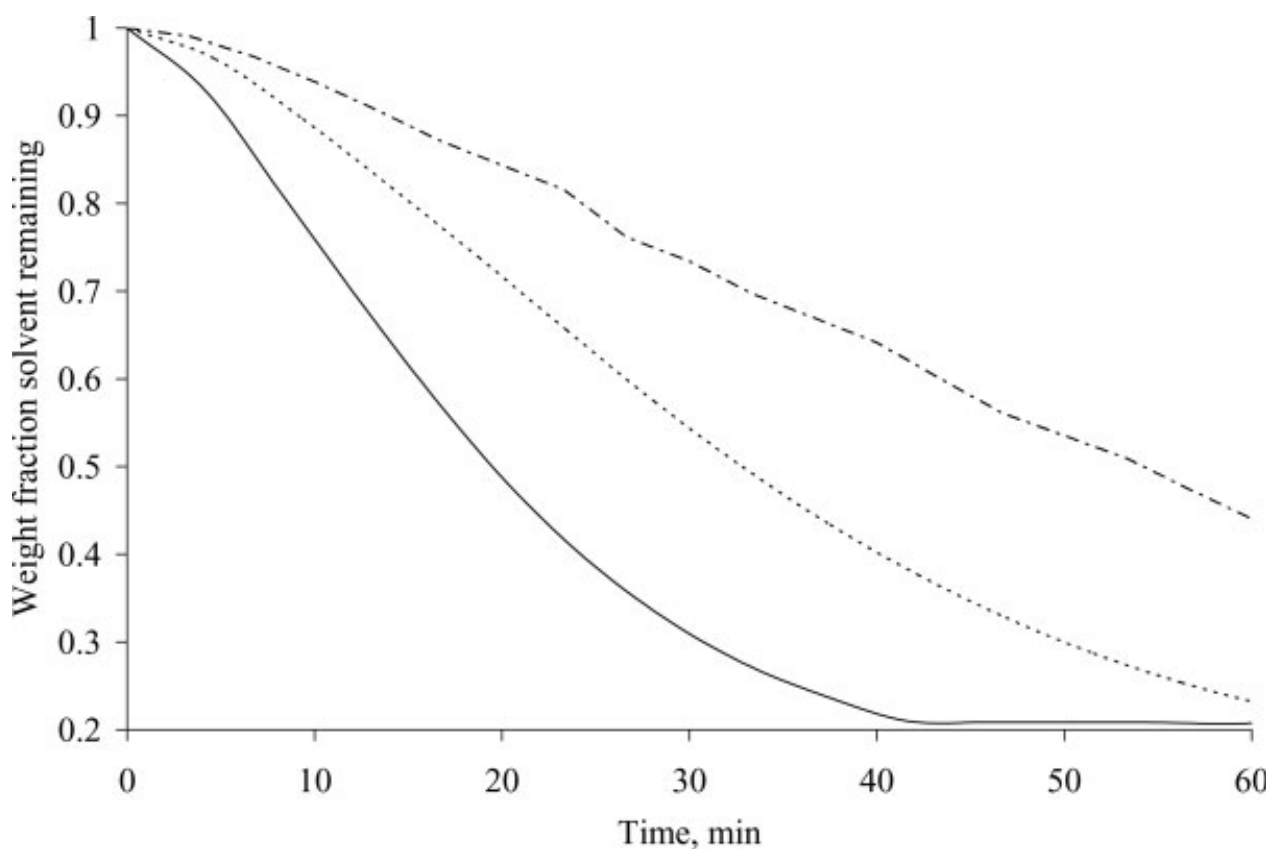

Figure 8. Total residual solvent volume fraction with various initial film thicknesses: (-) the remaining solvent volume fraction for an initial film thickness of $0.5 \mathrm{~mm},(.$.$) the volume fraction for an initial film thickness of 0.75 \mathrm{~mm}$, and $(\cdot-\cdot)$ the volume fraction for an initial film thickness of $1 \mathrm{~mm}$. 


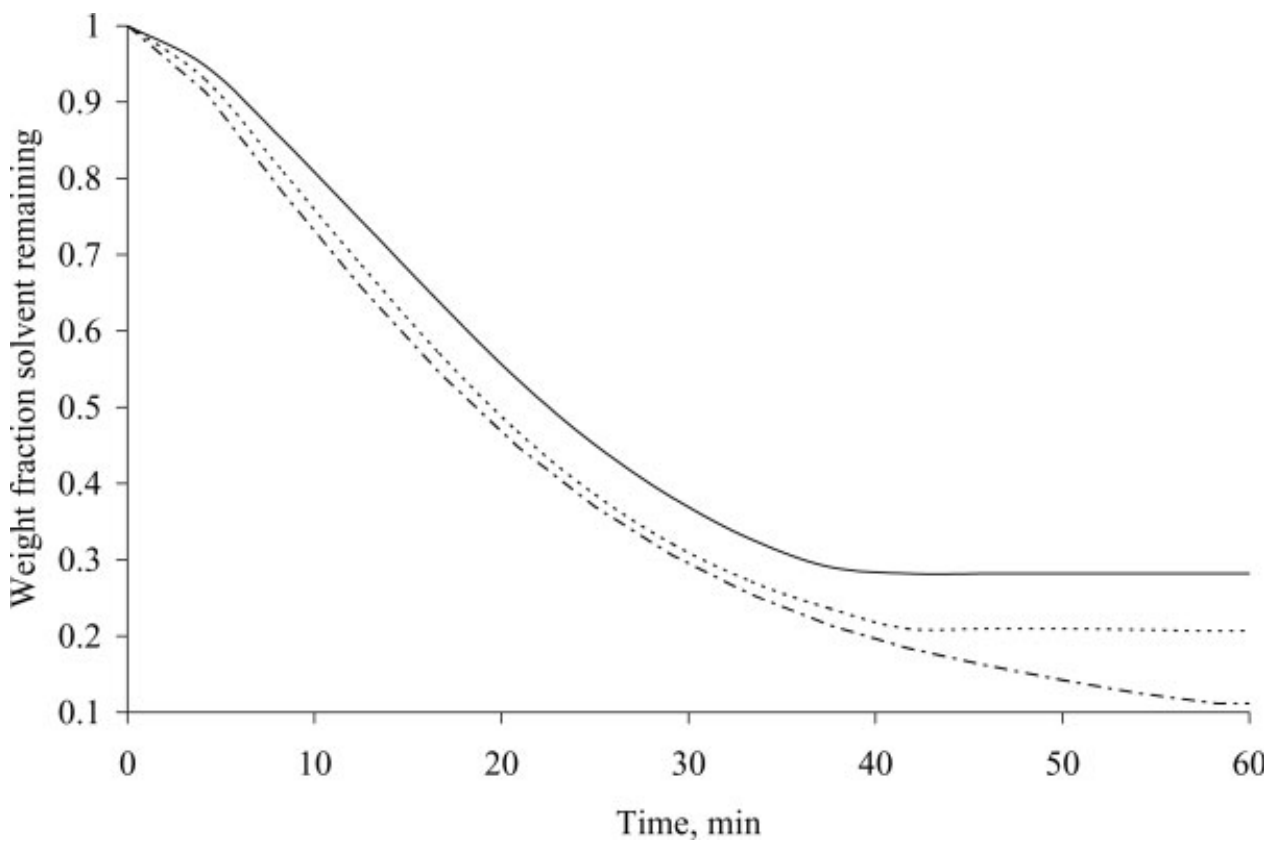

Figure 9. Effects of different water/methanol ratios on the total residual solvent volume fraction: the system behavior when the initial solvent ratio (water/methanol) is $(-) 2: 1,(.) 4:$.1 , and $(\cdot-\cdot) 8: 1$.

study the effect of the initial film thickness, three thicknesses $(0.5,0.75$, and $0.1 \mathrm{~mm})$ were used while other operating conditions were held constant. In Figure 8, we show that the increasing film thickness had a profound effect on the rate of solvent removal. Because the external conditions were held constant, we conclude that the differen- ces in these drying rates were purely due to diffusional resistances within the film. To further test the diffusional resistance of the film for the individual species, three different water/methanol concentrations were used. The simulation was repeated three times with different initial solvent concentrations (water/methanol $=2: 1,4: 1$, or $8: 1$ )

Table 4. Initial and Boundary Conditions for the PVA-Water-Methanol System for Comparison with Experimental Data ${ }^{\mathrm{a}}$

Initial Conditions

Temperature, $T_{0}$

Film thickness, $L_{0}$

Initial volume fraction of solvent $1, w_{10}$

Initial volume fraction of solvent $2, w_{20}$

Initial volume fraction of the crystalline polymer, $v_{0}$

Initial volume fraction of the amorphous polymer, $u_{0}$

Operating Conditions

Gas-polymer heat-transfer coefficient, $h^{\mathrm{G}}$

Gas-substrate heat-transfer coefficient, $h^{\mathrm{g}}$

Top-side air temperature, $T^{\mathrm{G}}$

Bottom-side air temperature, $T^{\mathrm{g}}$

Solvent 1 mass-transfer coefficient, $k_{i}^{\mathrm{G}}$

Solvent 2 mass-transfer coefficient, $k_{i}^{\mathrm{g}}$

Molar fraction of solvent 1 in gas

Molar fraction of solvent 2 in gas
$298 \mathrm{~K}$

$0.025 \mathrm{~cm}$

0.596

0.098

0.02

0.286

$0.10 \mathrm{~W} \mathrm{~cm}^{-2} \mathrm{~K}^{-1}$ $0.092 \mathrm{~W} \mathrm{~cm}^{-2} \mathrm{~K}^{-1}$

$298 \mathrm{~K}$

$298 \mathrm{~K}$

$1.7 \times 10^{-10} \mathrm{~s} \mathrm{~cm}^{-1}$ $2.3 \times 10^{-13} \mathrm{~s} \mathrm{~cm}^{-1}$

0

0

${ }^{\text {a }}$ Data taken from refs. 43 and 44. 


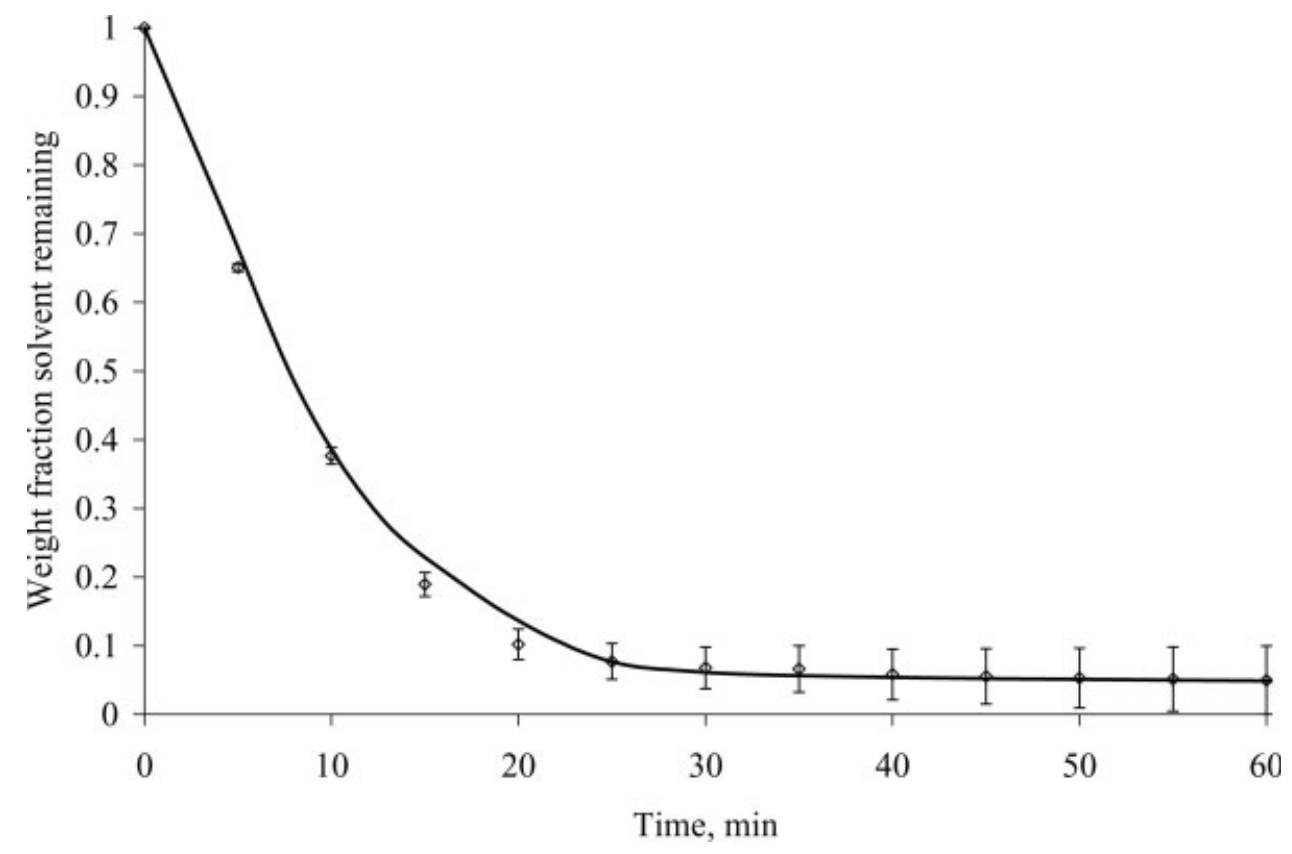

Figure 10. Comparison of $(\diamond)$ the experimental data and $(-)$ the simulation results of the solvent remaining during drying. The standard deviation of the experimental data was calculated with four repetitions of the experiment.

while the concentration of the polymer and other operating conditions were held constant. The results are shown in Figure 9. Clearly, the higher the initial water/methanol ratio was, the lower the final solvent residual level was. This can be attributed to the higher diffusivity of the water within the system in comparison with methanol. Comparing this with our previous model, which did not take into account glassy-rubbery transitions, we found that the difference in the initial solvent removal rate was considerable larger. We attribute this to the development of a glassy skin in the system, which slowed the diffusion of solvents further and gave rise to these differences in the initial solvent removal rate. In addition, the high concentration of water actually helped the diffusion of methanol in our system by creating more free volume. A further examination of the simulation data reveals that the development of the glassy skin may have prevented the slowly moving methanol from diffusing across the interface, thus trapping the solvent within the film and increasing the overall residual solvent amount.

To evaluate the performance of the model, we compared the operating conditions used in the model, as shown in Table 4, with our experimental procedures investigating the overall solvent removal rate. The initial conditions of the films were determined with the gravimetric methods described. Because the model was based on volume fractions whereas gravimetric experiments yield weight data, the weight percentage of the solvent remaining was used as a bridge to link these two techniques, and the data from the experiments and model were converted accordingly. The external mass-transfer coefficients were used as the fitting parameter for the simulation to correctly map the initial rate of solvent removal, which was then used to calculate the drying rates at later times. Figure 10 shows a direct comparison of the model predictions and experimental data. Overall, they were in good agreement with each other. The total residual solvent content was accurately predicted by the model, and this indicates that the diffusion model and the crystallization kinetics framework used were accurate.

This model represents our continued effort toward the understanding of the drying behavior in semicrystalline polymers. It explains and predicts several aspects of this complex problem, including the solvent removal rate, crystallization kinetics, and glassy-rubbery transitions. However, the effect of the glass-transition-temperature change of the polymer-solvent mixture due to solvent removal is not included in this model. In addition, we have used the same ther- 
modynamic theory for both rubbery and glassy regions. However, it needs to be modified for ternary glassy polymer-solvent mixtures. We will expand the model in the future to include those effects to paint a more complete picture of the drying behavior of glassy, semicrystalline polymers.

\section{CONCLUSIONS}

We have developed a mathematical model to investigate the development of glassy skin formation and its effect on the overall multicomponent drying behavior and crystallinity of semicrystalline polymers. Our model considered the drying behavior in rubbery and glassy regions separately to determine the effect of each region on the overall drying behavior. This was done with a new spatial coordinate to demarcate the two regions. Our study showed that the solvent diffusion behavior is significantly different in the two regions, and these differences affect the overall drying behavior of semicrystalline polymer films. This shows that the inclusion of the glassy region in the calculation is important in accurately predicting the behavior of semicrystalline polymer during drying. Parametric studies showed that the operating temperature, the film thickness, and the initial solvent ratios affect the drying behavior. The effects of the interaction between solvents as solvent-polymer interactions can be studied with this model to reveal relationships that are crucial to developing an optimum drying scheme. Our experimental results are in good agreement with our model predictions, and so this model can be used as a tool for the better design of drying systems.

The authors thank the National Science Foundation for its financial support through NSF-CTS0107168.

\section{REFERENCES AND NOTES}

1. Alsoy, S.; Duda, J. L. AIChE J 1999, 45, 896-905.

2. Alsoy, S.; Duda, J. L. Dry Technol 1998, 16, 1544.

3. Wong, S.-S.; Altinkaya, S. A.; Mallapragada, S. K. Polymer 2004, 45, 5151-5161.

4. Miltz, J.; Rosen-Doody, V. J Food Process Pres 1984, 8, 151-161.

5. Miltz, J.; Passay, N.; Mannheim, C. H. Packag Technol Sci 1992, 5, 49-56.
6. Nir, M. M.; Ram, A.; Miltz, J. Polym Eng Sci 1996, 36, 862-868.

7. Price, P. E., Jr.; Cairncross, R. A. Dry Technol 1999, 17, 1303-1311.

8. Errede, L. A.; Henrich, P. J.; Tiers, G. V. D. J Appl Polym Sci 1994, 54, 669-696.

9. Astarita, G.; Sarti, G. C. Polym Eng Sci 1978, 18, 388-395.

10. Vrentas, J. S.; Vrentas, C. M. J Appl Polym Sci 1996, 60, 1049-1055.

11. Cairncross, R. A.; Francis, L. F.; Scriven, L. E. Dry Technol 1992, 10, 893-923.

12. Cairncross, R. A.; Jeyadev, S.; Dunham, R. F.; Evans, K.; Francis, L. F.; Scriven, L. E. J Appl Polym Sci 1995, 58, 1279-1290.

13. Cairncross, R. A.; Durning, C. J. AIChE J 1996, 42, 2415-2425.

14. Cairncross, R. A.; Francis, L. F.; Scriven, L. E. AIChE J 1996, 42, 55-67.

15. Okazaki, M.; Shioda, K.; Masuda, K.; Toei, R. J Chem Eng Jpn 1974, 7, 99-105.

16. Yoshida, M.; Miyashita, H. Chem Eng J 2002, 86, 193-198.

17. Edwards, D. A. Macromol Theory Simul 1999, 8, 10-14.

18. Alsoy, S.; Duda, J. L. J Polym Sci Part B: Polym Phys 1999, 37, 1665-1675.

19. Zielinski, J. M.; Alsoy, S. J Polym Sci Part B: Polym Phys 2001, 39, 1496-1504.

20. Barr-Howell, B. D.; Gordon, E. J. Thermochim Acta 1991, 180, 147-154.

21. Price, P. E., Jr.; Cairncross, R. A. J Appl Polym Sci 2000, 78, 149-165.

22. Ngui, M. O.; Mallapragada, S. K. J Polym Sci Part B: Polym Phys 1998, 36, 2771-2780.

23. Ngui, M. O.; Mallapragada, S. K. J Appl Polym Sci 1999, 72, 1913-1920.

24. Ngui, M. O.; Mallapragada, S. K. Polymer 1999, 40, 5393-5400.

25. Vrentas, J. S.; Duda, J. L. J Appl Polym Sci 1980, 25, 1297-1310.

26. Vrentas, J. S.; Vrentas, C. M. Macromolecules 1991, 24, 2404-2412.

27. Wang, B.-G.; Yamaguchi, T.; Nakao, S.-I. J Polym Sci Part B: Polym Phys 2000, 38, 846856.

28. Romdhane, I. H.; Price, P. E., Jr.; Miller, C. A.; Benson, P. T.; Wang, S. Ind Eng Chem Res 2001, 40, 3065-3075.

29. Vrentas, J. S.; Vrentas, C. M. J Polym Sci Part B: Polym Phys 2004, 41, 785-788.

30. Ramesh, N. Ph.D. Thesis, Pennsylvania State University, University Park, PA, 2001.

31. Vrentas, J. S.; Duda, J. L. J Polym Sci Polym Phys Ed 1977, 15, 403-416.

32. Vrentas, J. S.; Duda, J. L. J Polym Sci Polym Phys Ed 1977, 15, 417-439.

33. Bearman, R. J. J Phys Chem 1961, 65, 19611968. 
34. Astarita, G.; Joshi, S. J Membr Sci 1978, 4, 165-182.

35. Flory, P. J. Principles of Polymer Chemistry; Cornell University Press: Ithaca, NY, 1953.

36. Favre, E.; Nguyen, Q. T.; Clement, R.; Neel, J. Eur Polym J 1996, 32, 303-309.

37. Duda, J. L.; Vrentas, J. S.; Ju, S. T.; Liu, H. T. AIChE J 1982, 28, 279-285.

38. Vrentas, J. S.; Duda, J. L.; Ling, H. C. J Polym Sci Polym Phys Ed 1985, 23, 275-288.

39. Zielinski, J. M.; Duda, J. L. AIChE J 1992, 38, 405-415.
40. Crank, J. Free and Moving Boundary Problems; Clarendon: Oxford, 1984.

41. Peppas, N. A.; Merrill, E. W. J Polym Sci Polym Chem Ed 1976, 14, 441-457.

42. Zielinski, J. M. Air Products, Inc., Allentown, PA. Personal communication, 2003

43. Perry's Chemical Engineer's Handbook; Perry, R. H.; Green, D. W.; Maloney, J. O., Eds.; McGrawHill: New York, 1997.

44. Polymer Handbook; Brandrup, J.; Immergut, E. H., Eds.; Wiley: New York, 1989. 